\title{
ON A RESULT OF NEHARI ${ }^{1}$
}

JAMES A. JENKINS

1. A few years ago Nehari [4] studied the situation of univalent functions mapping the exterior of the unit circle into a closed Riemann surface covering the sphere so that the point at infinity would go into a point on the surface covering the point at infinity, the function having the corresponding development

$$
z+a_{1} z^{-1}+a_{2} z^{-2}+\cdots
$$

He proved in particular that the region of variability of the coefficient $a_{1}$ is contained in a circle of radius 1 . His method was his potential theoretic version of the method of contour integration. In recent papers [2], [3] we have remarked that in a wide variety of situations the Area Principle leads to results superior to those obtained by the method of contour integration. It seems natural, therefore, to apply this principle to Nehari's problem and we find that the same is true here.

2. All the essential factors appear if we work with a general closed Riemann surface rather than specifying a covering surface. Indeed it is simply a matter of fixing a particular local uniformizing parameter at one point. The geometric structure of the covering surface intervenes only in relating such a local parameter to the form of development specified by Nehari. Even in his paper the case of a two-sheeted elliptic covering surface was simplified by a special choice through a preliminary normalization [4, p. 227]. Further, in the case of a surface of genus zero, the result is in principle trivial being equivalent to the familiar elementary result for the family $\Sigma[1$, p. 95]. Even for the two-sheeted covering surface of genus zero complete explicit results can be obtained in a simple elementary manner.

With these preliminaries we state the desired result in the following form.

TheOREM. Let $\Re$ be a closed Riemann surface of positive genus $g$. Let $A_{1}, A_{2}, \cdots, A_{g}, B_{1}, B_{2}, \cdots, B_{g}$ be a canonical homology basis of $\Re$. Let $\omega_{1}, \cdots, \omega_{0}$ be a basis for the differentials of the first kind on $\Re$ such that $\omega_{j}$ has respective $A$ and $B$ periods $\delta_{j k}, \mu_{j k}, k=1, \cdots, g$ where $\delta_{j k}$ is the familiar Kronecker $\delta$. Let $P$ be a fixed point of $\Re$ and let $z$ be a

Received by the editors June 28, 1965.

${ }^{1}$ Research supported in part by the National Science Foundation. 
local parameter representing $P$ as the point at infinity. Let $t^{*}$ be a differential of the second kind on $\Re$ regular apart from a double pole at $P$ at which point we have the representation

$$
t^{*}(z) d z=\left(1+b_{2} z^{-2}+\text { higher powers of } z^{-1}\right) d z
$$

and having zero $A$ periods and $B$ periods $\pi_{j+g}, j=1, \cdots, g$. Let $f$ be a conformal mapping of $|w|>1$ into $\Re$ carrying the point at infinity into $P$ and having there in terms of the chosen local parameter the representation

$$
f(w)=w+a_{0}+a_{1} w^{-1}+\text { higher powers of } w^{-1} .
$$

Let the matrix $\left(n_{i j}\right)$ be the $g \times g$ matrix inverse to $\left(\Im \mu_{j k}\right)$. Then the region of values of the coefficient $a_{1}$ lies in a circle of radius

$$
\left[1-\frac{1}{4 \pi} \sum_{j=1}^{o} \sum_{k=1}^{o} n_{j k} \pi_{j+g} \bar{\pi}_{k+g}\right]^{1 / 2} .
$$

It should be observed that not all periods $\pi_{j+g}, j=1, \cdots, g$, can be zero since that would make the integral of $t^{*}(z) d z$ on $\Re$ a meromorphic function with just one simple pole.

We form the differential of the second kind

$$
t d z=\left(t^{*}+\sum_{j=1}^{g} \alpha_{j} \omega_{j}\right) d z .
$$

Consider the image $I_{r}$ on $\Re$ under $f$ of $|w|>r, r>1$, and the image $\Gamma_{r}$ of $|w|=r$ (sensed to agree with the positive sense of the latter). In the complement of $I_{r}$ we can find simple closed contours $a_{j}, b_{j}$, $j=1, \cdots, g$, which have altogether one common point $Q$ and whose deletion from $\Re$ reduces the latter to a simply-connected domain $\mathfrak{D}$ and which appear on the boundary of $\mathfrak{D}$ in positive order as $a_{1}, b_{1}, a_{1}^{-1}, b_{1}^{-1}, \cdots, a_{g}, b_{g}, a_{g}^{-1}, b_{g}^{-1}$. We denote this contour by $\Lambda$ and the domain on $\Re$ bounded by $\Gamma_{r}$ and $\Lambda$ by $\Delta_{r}$. Then

$$
\iint_{\Delta_{r}}|t(\zeta)|^{2} d A_{\zeta} \geqq 0
$$

where $d A_{\zeta}$ denotes the element of area in terms of the generic local uniformizing parameter $\zeta$ on $\Re$. In $\mathfrak{D}$ we can integrate $t(\zeta) d \zeta$ to obtain a meromorphic function $T$ which will have expansion about $P$ in terms of the local parameter $z$

$$
T(z)=z+c_{1} z^{-1}+\text { higher powers of } z^{-1} .
$$

Inequality (1) can be rewritten as 


$$
\frac{1}{2 i} \int_{\Gamma_{r}} \bar{T}(\zeta) t(\zeta) d \zeta+\frac{1}{2 i} \int_{\Delta} \bar{T}(\zeta) t(\zeta) d \zeta \geqq 0 .
$$

Since the $A$ periods of $t$ are $\alpha_{k}, k=1, \cdots, g$, and the $B$ periods $\pi_{k+o}+\sum_{j=1}^{o} \alpha_{j} \mu_{j k}$ we find

$$
\begin{aligned}
& \frac{1}{2 i} \int_{\Delta} \bar{T}(\zeta) t(\zeta) d \zeta \\
&=\frac{1}{2 i} \sum_{k=1}^{g}\left[\bar{\alpha}_{k}\left(\pi_{k+\vartheta}+\sum_{j=1}^{g} \alpha_{j} \mu_{j k}\right)-\alpha_{k}\left(\bar{\pi}_{k+o}+\sum_{j=1}^{g} \bar{\alpha}_{j} \bar{\mu}_{j k}\right)\right] .
\end{aligned}
$$

On the other hand

$$
\frac{1}{2 i} \int_{\Gamma_{r}} \bar{T}(\zeta) t(\zeta) d \zeta=\frac{1}{2 i} \int_{|w|=r} \bar{T}(f(w)) d T(f(w))
$$

where $T(f(w))$ is regular in $|w|>1$ apart from a simple pole at the point at infinity and is represented there by the expansion

$$
T(f(w))=w+a_{0}+\left(a_{1}+c_{1}\right) w^{-1}+\text { higher powers of } w^{-1} .
$$

The integral on the right hand side of (3) is thus equal to

$$
\pi\left(r^{2}-\left|a_{1}+c_{1}\right|^{2} r^{-2}+\text { higher powers of } r^{-1}\right) .
$$

Letting $r$ tend to 1 we have from (2) by the usual argument

$$
\begin{aligned}
& \left|a_{1}+c_{1}\right|^{2} \\
& \leqq 1+\frac{\pi^{-1}}{2 i} \sum_{k=1}^{g}\left[\bar{\alpha}_{k}\left(\pi_{k+o}+\sum_{j=1}^{o} \alpha_{j} \mu_{j k}\right)-\alpha_{k}\left(\bar{\pi}_{k+\vartheta}+\sum_{j=1}^{o} \bar{\alpha}_{j} \bar{\mu}_{j k}\right)\right] .
\end{aligned}
$$

The right hand side of (4) reduces to

$$
1-\pi^{-1} \sum_{k=1}^{0} \Im\left(\bar{\pi}_{k+\rho} \alpha_{k}\right)+\pi^{-1} \sum_{j=1}^{0} \sum_{k=1}^{0}\left(\Im \mu_{j k}\right) \alpha_{j} \bar{\alpha}_{k}
$$

where we use the well known fact that $\mu_{j k}$ is symmetric in $j$ and $k$. We recall also that the matrix $\left(\Im \mu_{j k}\right)$ is positive definite, thus nonsingular, so has an inverse $\left(n_{i j}\right)$. We readily verify that the expression (5) is minimized by the choice

$$
\alpha_{k}=\frac{1}{2} i \sum_{j=1}^{g} n_{k j} \pi_{j+\theta}
$$

and that this minimal value is 


$$
1-\frac{1}{4 \pi} \sum_{j=1}^{g} \sum_{k=1}^{g} n_{j k} \pi_{j+g} \bar{\pi}_{k+g} .
$$

This completes the proof of our theorem.

We observe that the quantity (6) is always strictly less than 1. Hence for a surface of positive genus the value 1 for the radius given by Nehari is never best possible. (He said that it was not "in general.")

3. In the particular case of genus one we can interpret the theorem as applying to functions regular and univalent in the unit circle and not taking any two values congruent with respect to a pair of periods $2 \omega_{1}, 2 \omega_{2}$ (which we may take to satisfy $\Im\left(\omega_{1}^{-1} \omega_{2}\right)>0$ ). In particular we will take the distinguished point to correspond to the origin $Z=0$, the assigned local parameter being $z=Z^{-1}$. We see then we can use for $t^{*}$ the differential

$$
-\left(\varphi(Z)+\eta_{1} \omega_{1}^{-1}\right) d Z
$$

where $\varnothing$ denotes the Weierstrass $\&$-function and $2 \eta_{1}, 2 \eta_{2}$ are the additive terms for the corresponding $\zeta$-function. Its periods are $0,-\pi i \omega_{1}^{-1}$ and its expansion in terms of $z$ is

$$
\left(1+\eta_{1} \omega_{1}^{-1} z^{-2}+\cdots\right) d z .
$$

The only differential of the first kind, normalized as above, is $\left(2 \omega_{1}\right)^{-1} d Z$ with periods $1, \omega_{1}^{-1} \omega_{2}$ and the matrix reduces to a single element $\left(\Im \mu_{11}\right)=\left(\Im\left(\omega_{1}^{-1} \omega_{2}\right)\right)$. Similarly we have $\left(n_{11}\right)=\left(1 / \Im\left(\omega_{1}^{-1} \omega_{2}\right)\right)$. Thus the choice of $\alpha_{1}$ corresponding to the extremal situation is

$$
\alpha_{1}=2 \pi \bar{\omega}_{1} A^{-1}
$$

where $A$ denotes the area of a period parallelogram. Further the corresponding differential $t d z$ is seen by an easy calculation to have the form

$$
\left(-\wp(Z)+2 i\left(\eta_{2} \bar{\omega}_{1}-\eta_{1} \bar{\omega}_{2}\right) A^{-1}\right) d Z .
$$

Thus its expansion in terms of $z$ is

$$
\left(1-2 i\left(\eta_{2} \bar{\omega}_{1}-\eta_{1} \bar{\omega}_{2}\right) A^{-1} z^{-2}+\cdots\right) d z
$$

and that for the corresponding function $T$ is

$$
z+2 i\left(\eta_{2} \bar{\omega}_{1}-\eta_{1} \bar{\omega}_{2}\right) A^{-1} z^{-1}+\cdots .
$$

Finally, if the function $f$ is regular and univalent for $|W|<1$ with expansion at the origin

$$
W+A_{2} W^{2}+A_{3} W^{3}+\cdots,
$$


the expansion of $1 / f$ in terms of $w=W^{-1}$ is

$$
w-A_{2}+\left(A_{2}^{2}-A_{3}\right) w^{-1}+\cdots .
$$

In this particular case we then have the result:

COROLlary. Let $f(W)$ be regular and univalent for $|W|<1$ with expansion about $W=0$

$$
W+A_{2} W^{2}+A_{3} W^{3}+\cdots
$$

and take no two values congruent with respect to a pair of periods $2 \omega_{1}$, $2 \omega_{2}$ (satisfying $\left.\Im\left(\omega_{1}^{-1} \omega_{2}\right)>0\right)$. Then

$$
\left|A_{2}^{2}-A_{3}+2 i\left(\eta_{2} \bar{\omega}_{1}-\eta_{1} \bar{\omega}_{2}\right)\right| \leqq\left(1-\pi A^{-1}\right)^{1 / 2}
$$

where $2 \eta_{1}, 2 \eta_{2}$ are the additive terms for the $\zeta$-function with the given periods and $A$ is the area of a period parallelogram.

\section{BIBLIOGRAPH Y}

1. James A. Jenkins, Univalent functions and conformal mapping, Springer, Berlin, 1958.

2. - Some area theorems and a special coefficient theorem, Illinois J. Math. 8 (1964), 80-99.

3. - On Bieberbach-Eilenberg functions. III, Trans. Amer. Math. Soc. 119 (1965), 195-215.

4. Zeev Nehari, On the coefficients of $R$-univalent functions, Duke Math. J. 22 (1955), 223-227.

WASHINGTON UNIVERSITY 